\section{MS42-P3 News from the Swiss Spallation \\ Neutron Source SINQ: Diffraction at Non-Ambient Conditions}

Christian Rüegg ${ }^{1,2}$, Romain Sibille ${ }^{1}$, Jürg Schefer ${ }^{1}$, Vladimir Pomjakushin ${ }^{1}$, Denis Sheptyakov ${ }^{1}$, Lukas Keller ${ }^{1}$, Emmanuel Canevet ${ }^{1}$, Tobias Panzner ${ }^{1}$, Oksana Zaharko ${ }^{1}$

1. Laboratory for Neutron Scattering and Imaging, Paul Scherrer Institute

2. Department of Quantum Matter Physics, University of Geneva

email: christian.rueegg@psi.ch

We present news from the Swiss Spallation Neutron Source SINQ and its instruments for neutron diffraction. The neutron diffraction group operates four instruments: the single-crystal diffractometer ZEBRA, the two powder diffractometers HRPT and DMC, and the strain scanner POLDI as shown in Fig. 1.

The new neutron single-crystal diffractometer ZEBRA, which replaces the TriCS instrument, is now in commissioning phase. It will allow crystallographic and parametric studies (temperature, magnetic and electric fields, pressure). ZEBRA is designed to achieve high peak-to-background ratio and to operate in high magnetic fields up to 11 Tesla. The instrument is aimed at resolving challenges emerging in systems that are available as small crystals only and requiring extreme sample environments.

The applications of the thermal high-resolution multi-detector powder diffractometer HRPT are high-resolution refinements of chemical and magnetic structures as well as phase analysis of novel materials. Recently HRPT was equipped with computer controlled sample changers of eight (RT) and five (1.7-310 K) samples with sample rotation, which significantly increases the efficiency of operation. The exclusive stroboscopic mode allows measurements of crystal structures as a function of time with time resolution down to $10 \mathrm{~ms}$.

The cold neutron powder diffractometer DMC is complementary to HRPT and ZEBRA and is designed for high-intensity measurements of weak magnetic intensities. Its recent success in mapping the reciprocal space of single crystals prompts the development of a new high-efficiency two-dimensional neutron detector, which will be available in the near future. A new non-magnetic sample table allows fields up to 6 Tesla.
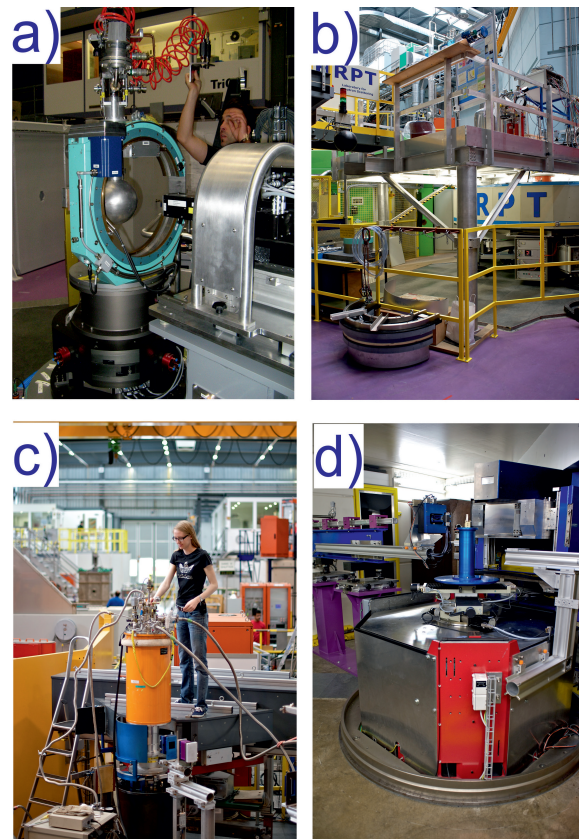

Figure 1. Diffraction instruments at the Swiss Spallation Neutron Source SINQ: a) ZEBRA, b) HRPT, c) DMC and d) POLDI.

Keywords: neutron diffraction, instrumentation, pressure, magnetic fields 